\title{
Collaborative Policy of the Supply-Hub for Assemble-to-Order Systems with Delivery Uncertainty
}

\author{
Guo Li, ${ }^{1}$ Mengqi Liu, ${ }^{2}$ Xu Guan, ${ }^{3}$ and Zheng Huang ${ }^{4}$ \\ ${ }^{1}$ School of Management and Economics, Beijing Institute of Technology, Beijing 100081, China \\ ${ }^{2}$ School of Business Administration, Hunan University, Changsha 410082, China \\ ${ }^{3}$ Economics and Management School, Wuhan University, Wuhan 430072, China \\ ${ }^{4}$ School of Management, Huazhong University of Science and Technology, Wuhan 430074, China
}

Correspondence should be addressed to Mengqi Liu; 1069679071@qq.com

Received 26 July 2013; Revised 6 March 2014; Accepted 13 March 2014; Published 29 May 2014

Academic Editor: Tinggui Chen

Copyright (C) 2014 Guo Li et al. This is an open access article distributed under the Creative Commons Attribution License, which permits unrestricted use, distribution, and reproduction in any medium, provided the original work is properly cited.

\begin{abstract}
This paper considers the collaborative mechanisms of the Supply-Hub in the Assemble-to-Order system (ATO system hereafter) with upstream delivery uncertainty. We first propose a collaborative replenishment mechanism in the ATO system, and construct a replenishment model with delivery uncertainty in use of the Supply-Hub. After transforming the original model into a onedimensional optimization problem, we derive the optimal assembly quantity and reorder point of each component. In order to enable the Supply-Hub to conduct collaborative replenishment with each supplier, the punishment and reward mechanisms are proposed. The numerical analysis illustrates that service level of the Supply-Hub is an increasing function of both punishment and reward factors. Therefore, by adjusting the two factors, suppliers' incentives of collaborative replenishment can be significantly enhanced, and then the service level of whole ATO system can be improved.
\end{abstract}

\section{Introduction}

The supply disruptions in ATO systems caused by upstream suppliers nowadays happen frequently due to the influences of natural disasters, strikes, terrorist attacks, political instability, and other factors. As shown by Li et al. [1, 17], in 2000 Philips Semiconductor Factory's fire led to Ericsson's supply disruption of the chip, which caused Ericsson a loss of 1.8 billion dollars and $4 \%$ of its market share. In July 2010, Hitachi's unexpected shortage of car engine control part resulted in the shutdown of Nissan's plant for 3 days, and the production of 1.5 million cars was affected by this. In March 2011, Japan's 9-magnitude earthquake in northeast devastated the industrial zone. Three major automakers in Japan, Toyota, Honda and Nissan, were affected by supply disruptions, and some Sino-Japanese joint ventures in China also had different levels of supply disruptions. Accordingly, driven by these serious losses caused by supply uncertainty, both scholars and practitioners try to find out effective ways to improve ATO systems' overall performances by handling upstream disruptions. Under this circumstance, Supply-Hub arises.

Supply-Hub, also called VMI (vendor-managed inventory) Hub, is often located near the core manufacturer to integrate the logistics operation of part or all suppliers and mostly managed by the third party logistics operator. Supply-Hub operation mode evolves from the traditional VMI operation mode. In practice, because there exist all sorts of problems in the distributed VMI operation mode, some advanced core manufacturers consider to manage independent warehouses in a centralized way instead of the original decentralized way through resources integration, organization reconstruction, and coordination optimization. This not only helps to reduce the investment cost in fixed facilities, but also can greatly reduce the operation and management cost of the whole supply chain. Gradually, a lot of the third party logistics distribution centers, which mainly focus on integration management service of upstream supply logistics, appear. 
In this sense, the Supply-Hub can be viewed as an intermediary between the suppliers and manufacturer, and Indirect Distribution Channel is the intermediary between the manufacturer and retailers. Furthermore, the SupplyHub can reduce the risk of components shortage caused by desynchronized delivery from different suppliers and improve the efficiency and benefit of supply chain. Nonetheless, although there exist some papers that take the SupplyHub into consideration, how to coordinate the suppliers by useful policies for the Supply-Hub is still rarely examined explicitly.

To address the gap between the practice and current literature, we investigate the interaction between delivery uncertainty and coordinative policy of the Supply-Hub and mainly address the following questions:

(1) What are the optimal replenishment decisions for the Supply-Hub in ATO systems with multiple suppliers and one manufacturer in case of uncertain delivery time?

(2) How would the Supply-Hub coordinate the suppliers, eliminate the delivery uncertainty, and improve the whole service level?

(3) What are the relations between the two coordinative factors and service level of the Supply-Hub?

To answer these questions, we consider an ATO system with multiple suppliers, one Supply-Hub and one manufacturer. This paper aims to establish a cost model with consideration of the effects caused by each component's delivery time that may be sooner or later than the expected arrival time. The reorder point of each component and assembly quantity are regarded as the decision variables, and we propose an order policy that minimizes the supply chain's total cost. Since the model in this paper can be viewed as a convex programming problem, we provide the unique optimal solution. Finally, we apply the punishment and reward mechanisms to the SupplyHub for the purpose of coordinating suppliers and improving service level, and through theoretical and numerical analysis we find the relations between the two coordinative factors and service level.

\section{Literature Review}

Production uncertainty can be attributed to the uncertainty of demand and supply. In recent years, some scholars investigate some secondary factors that cause supply delay in ATO systems, such as Song et al. [2, 3], Lu et al. [4, 5], Hsu et al. [6], Xu and Li [7], Plambeck and Ward [8, 9], Li and Wang [10], Lu et al. [11], Doğru et al. [12], Hoena at al. [13], Bernstein et al. [14],Reiman and Wang [15], Bušić et al. [16], and Li et al. $[1,17]$.

Song and Yao [3] consider the demand uncertainty in ATO systems under random lead time and expand random lead time into an inventory system assembled by a number of components. By assuming that the demand obeys passion distribution and that the arriving time of different components is independent and identically distributed, they conclude that the bigger the mean of lead time of components is, the higher the safety stock should be set, and they also give definite methods of finding the optimal safety stock under certain constraint of service level. Based on this, Lu and Song [5] consider the inventory system where products are composed of many components with random lead time. They deduce the optimal values that the safety stock should be set under different means of lead time. Hsu et al. [6] explore optimal inventory decision making in ATO systems in the situation that the demand is random, and the cost and lead time of components are sensitive to order quantity. Li and Wang [10] focus on the inventory optimization in a decentralized assembly system where there exists competition among suppliers under random demand and sensitive price. Hoena et al. [13] explore ATO systems with multiple end-products. They devide the system into several subsystems which can be analyzed independently. Each subsystem can be approximated by a system with exponentially distributed lead time, for which an exact evaluation exists. Bušić et al. [16] present a new bounding method for Markov chains inspired by Markov reward theory. With applications to ATO systems, they construct bounds by redirecting selected sets of transitions, facilitating an intuitive interpretation of the modifications of the original system. Li et al. $[1,17]$ consider an assembly system with two suppliers and one manufacturer under uncertainty delivery time. They prove that a unique Nash equilibrium exists between two suppliers. Decroix et al. [18] consider the inventory optimization problem in ATO systems where the product demand is random and components can be remanufactured. All literature above consider some optimal problems, such as stocks or order quantities in ATO systems from different perspectives. And the common characteristics are as follows: (1) the views expressed in these works are based on a single assembly manufacturing enterprise, rather than the whole supply chain. (2) Most papers assume that the replenishment of components is based on make-to-stock environment, rather than JIT replenishment. Therefore, how to realize the two-dimensional collaborative replenishment of multiple suppliers in JIT replenishment mode is the most urgent problem that needs to be solved currently.

Zimmer [19] studies the supply chain coordination between one manufacturer and multiple suppliers under uncertain delivery. In the worst case, under decentralized decision the optimal decisions of the manufacturer and suppliers are analyzed and in the best situation, under symmetric information, the optimal decision of supply chain is also obtained. Two kinds of coordination mechanisms (punishment and reward) are established, which realize the flexible cost allocation between collaborative enterprises. In the works of Gurnani [20],Gurnani and Gerchak [21], and Gurnani and Shi [22], the two-echelon supply chain is composed of two suppliers and one manufacturer. Under uncertain delivery quantity caused by suppliers' random yield, each side is optimized in decentralized and centralized decision and the total cost of supply chain is lower in centralized decision compared to the decentralized. Finally, the collaborative contract is proposed to coordinate the suppliers and manufacturer. In fact, the above articles study the assembly system based on the whole supply chain under 


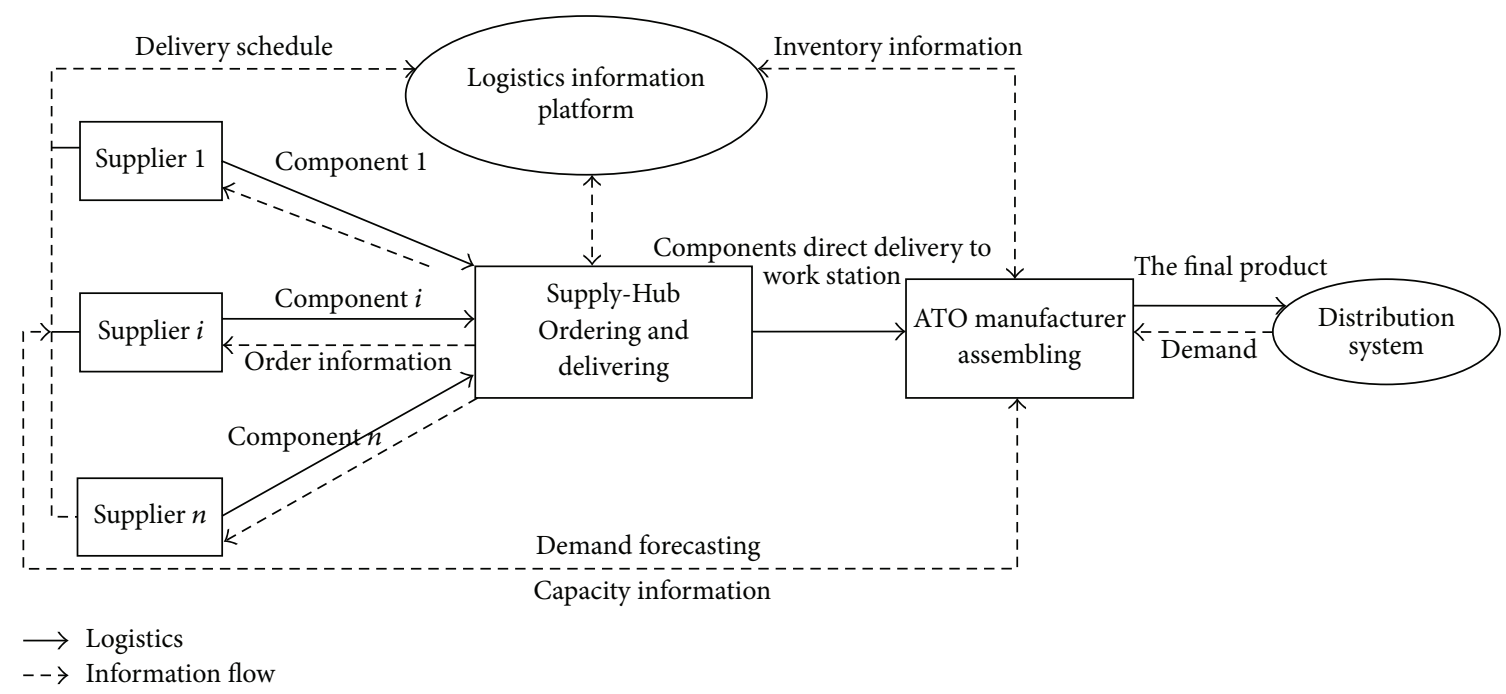

FIGURE 1: Framework of logistics and information flow based on the Supply-Hub.

random delivery quantity, but they do not take the random delivery time into consideration.

As to the Supply-Hub, Barnes et al. [23] find that Supply$\mathrm{Hub}$ is an innovative strategy to reduce cost and improve responsiveness used by some industries, especially in the electronics industry, and it is a reflection of delaying procurement. Firstly they give the definition of Supply-Hub and review its development, then they propose a prerequisite to establish a Supply-Hub and come up with a way to operate it. Shah and Goh [24] explore the operation strategy of the Supply-Hub to achieve the joint operation management between customers and their upstream suppliers. Moreover, they discuss how to manage the supply chain better in vendor-managed inventory model, and find that the relation between operation strategy and performance evaluation of the Supply-Hub is complex and nonlinear. As a result, they propose a hierarchical structure to help the Supply-Hub achieve the balance among supply chain members.

Based on the Supply-Hub, Ma and Gong [25] develop, respectively, collaborative decision-making models of production and distribution with considering the matching of distribution quantity between suppliers. The result shows that, the total cost of supply chain and production cost of suppliers decrease significantly, but the logistics cost of manufacturers and cost of Supply-Hub operators increase. With the consideration that multiple suppliers provide different components to a manufacturer based on the Supply-Hub, Gui and $\mathrm{Ma}$ [26] establish an economical order quantity model in such two ways as picking up separately from different suppliers and milk-run picking up. The result shows that the sensitivity to carriage quantity of the transportation cost per unit weight of components and the demand variance in different components have an influence on the choices of the two picking up ways. Li et al. [27] propose a horizontally dualsourcing policy to coordinate the Supply-Hub model. They indicate that the total cost of supply chain can be decreased obviously while the service level will not be reduced by using this horizontally collaborative replenishment policy.
However, how the Supply-Hub plays with the consolidation function is rarely discussed in detail, for example, how to improve the service level of upstream assembly system and efficiency of the whole supply chain. This issue is of great practical significance, because a wrong decision of certain component's replenishment will make the right decisions of other components' replenishment in the same Bill of Material (BOM) nonsense, thus leading to the low efficiency of the whole supply chain [27]. After introducing the BOM into consideration of order policy, due to the matching attribution of all materials, calculations of optimal reorder point of each component and assembly quantity are very complex, so we transform the original model into a one dimensional optimization problem and successfully obtain the optimal values of the decision variables. After that we propose a collaborative policy of the Supply-Hub for ATO systems with delivery uncertainty.

\section{Model Description and Formulation}

3.1. Model Assumptions. The operation framework of this model is illustrated in Figure 1 [25]. Based on this, we propose the following assumptions.

(1) According to the BOM, the manufacturer needs $n$ different kinds of components to produce the final product and each supplier provides one kind of the components, with the premise that the delivery quantity of each component should meet the equation Item 1 : Item $2: \ldots$ : Item $n=1$ : $1: \cdots: 1$. The manufacturer entrusts the Supply-Hub to be in charge of the JIT ordering and delivery service. The supply chain is an ATO system which consists of $n$ suppliers, one manufacturer, and one Supply-Hub. Note that the model in this paper only considers the cost of the two-echelon supply chain that includes the Supply-Hub and manufacturer and omits the suppliers' costs.

(2) The time spent by the manufacture for assembling the components is assumed to be 0 , which is appropriate 


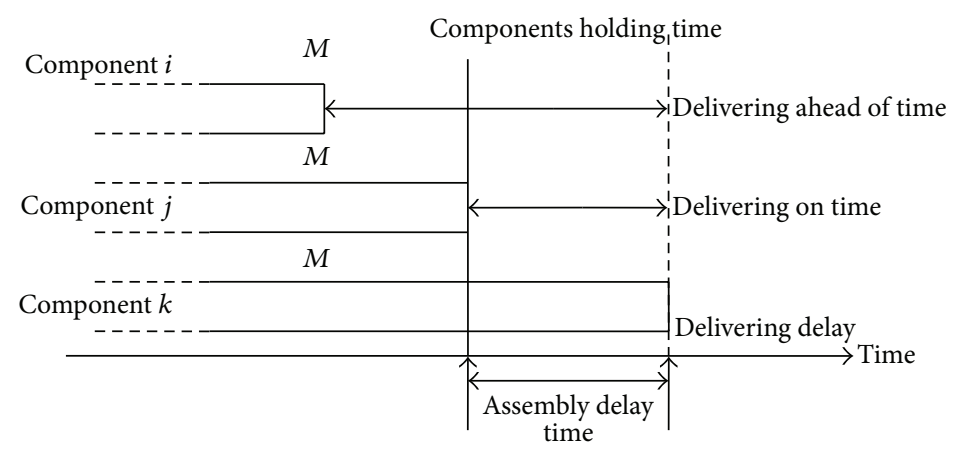

FIgURE 2: Arrival situations of different components in the Supply-Hub.

when the suppliers are relatively far away from each other. In addition, When the inventory of the final product turns to be used up, the manufacturer begins to assemble the components, and we call it the starting point of the assembly. So in the whole ordering and delivery process, there are two more situations in the Supply-Hub besides all components' arriving on time: (1) if the components arrive sooner than the expected assembly starting point, the Supply-Hub has to hold components until the manufacturer's inventory is used up. In this situation, the holding cost of all components is $\sum_{i=1}^{n} \mathrm{TC}_{h_{i}}$. (2) In another situation, because the Supply-Hub has to wait for all components' arrival, if there is a delay delivery of certain component, the assembly time will be delayed, resulting in the shortage cost $\mathrm{TC}_{\pi}$ (see Figure 2).

(3) The Supply-Hub delivers components to the manufacturer in certain frequency, such as $K$ times, then Order Quantity $=k \times$ delivery quantity [28]. It may be assumed here that $k=1$, and the Supply-Hub adopts the lot-for-lot method to distribute the components. If the manufacturer needs to assemble $Q$ final products, the Supply-Hub needs to order $Q$ components from each supplier. The lead time $Y_{i}(i=1,2 \ldots, n)$ of the components is mutually independent random variables, and the probability distribution function and probability density function are, respectively, $F_{i}(x)$ and $f_{i}(x)$.

(4) The market demand $D$ per unit time for the final product is fixed, and the backorder policy is adopted to deal with shortage. Without loss of generality, we assume $\pi>h>$ $\sum_{i=1}^{n} h_{i}$.

Related parameters are defined as follows.

$A$ is unit order cost of components.

$\pi$ is unit shortage cost of the final product.

$Y_{i}$ is random lead time of component $i$.

$L_{i}$ is late or early arrival period of component $i$.

$h_{i}$ is unit holding cost of component $i$.

$h$ is unit holding cost of the final product.

$r_{i}$ is reorder point of component $i$ (decision variables).

$R_{i}$ is distribution parameter of lead time of component $i$.

$Q$ is assembly quantity (decision variable).

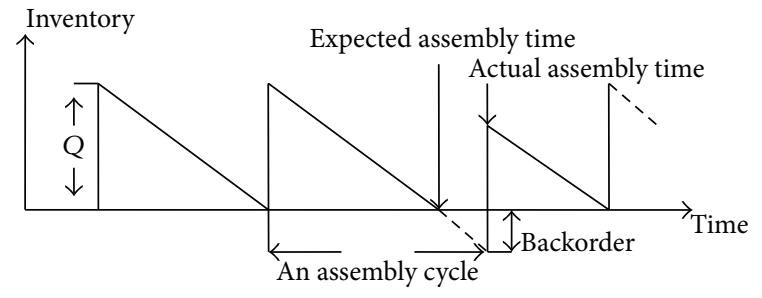

FIGURE 3: Inventory status of the manufacturer's final product.

3.2. Model Formulation. The period between two adjacent actual assembly starting points can be regarded as a cycle (see Figure 3). In each cycle, the Supply-Hub needs to deliver a collection of $Q$ components directly to the manufacturer's work station. Besides, the purchase order should be issued before the expected assembly starting point, which should be issued at the moment of $r_{i} / D$. The late or early arrival period of each component can be expressed as the difference between actual lead time and expected lead time, or $L_{i} \equiv Y_{i}-$ $r_{i} / D$. In addition, we define $L_{i}^{+} \equiv \max \left\{L_{i}, 0\right\}$, which means the delay time of component $i$, and $L \equiv \max _{1 \leq i \leq n}\left\{L_{i}^{+}\right\} \equiv$ $\max _{1 \leq i \leq n}\left\{L_{i}, 0\right\}$, which means the delay time of the manufacturer's assembling. If $L_{i}$ is negative, it means component $i$ is delivered before the expected assembly starting point.

Based on the assumptions and definitions above, we can derive the following conclusions.

(1) Average delay time of the manufacturer's assembling per cycle is $E[L]$.

(2) Average shortage quantity of the final product for the manufacturer is $D \cdot E^{2}[L] / 2$ (as shown in Figure 4).

(3) Average holding cost of the final product for the manufacturer per cycle is $(h / 2 D)(Q-D \cdot E[L])^{2}$.

(4) Average holding time of component $i$ for the SupplyHub per cycle is $[L]-E\left[L_{i}\right]$.

Therefore, for the two-echelon supply chain model that consists of the Supply-Hub and manufacturer, the average total cost $\mathrm{TC}_{p}$ per cycle is the sum of the order cost of components, holding cost of the components, holding cost 


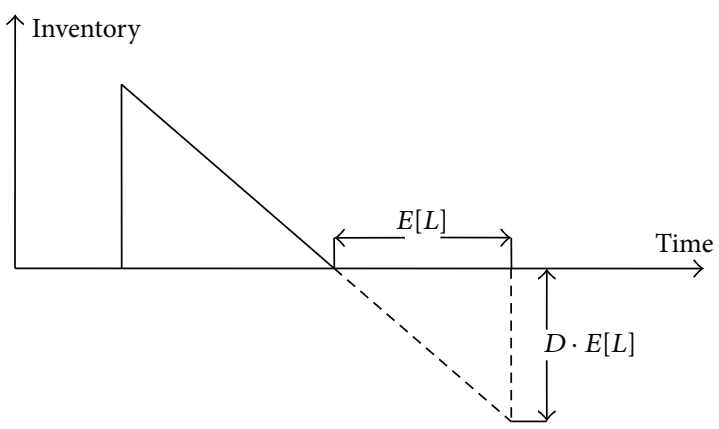

FIGURE 4: Shortage status of the manufacturer' final product.

and shortage cost of the final product, which can be described as follows:

$$
\begin{aligned}
\mathrm{TC}_{p}= & A+\frac{h}{2 D}(Q-D \cdot E[L])^{2}+Q \sum_{i=1}^{n} h_{i}\left(E[L]-E\left[L_{i}\right]\right) \\
& +\frac{\pi D \cdot E^{2}[L]}{2} .
\end{aligned}
$$

Furthermore, the delivery frequency is $D / Q$, so the average total cost per unit time is $\mathrm{TC}(Q, r)$, which can be calculated by the following formula:

$$
\begin{aligned}
\mathrm{TC}(Q, r)= & \frac{A D}{Q}+\frac{h Q}{2}+D\left(\sum_{i=1}^{n} h_{i}-h\right) E[L] \\
& +\frac{(h+\pi) D^{2}}{2 Q} E^{2}[L]-D \sum_{i=1}^{n} h_{i} E\left[L_{i}\right] .
\end{aligned}
$$

In general, the model in the paper can be abstracted as the following nonlinear programming problem $(P)$ :

$$
\begin{array}{cc}
\text { Min } \quad \mathrm{TC}(Q, r) \\
\text { s.t. } \quad Q \geq 0, \quad r_{i} \geq 0, \\
\\
\quad i=1,2, \ldots, n .
\end{array}
$$

\section{Model Analysis and Solution}

4.1. Model Analysis. To derive the results, we first take the partial derivatives of TC $(Q, r)$ with respect to $Q$ and $r_{i}(i=$ $1,2, \ldots, n)$ :

$$
\begin{gathered}
\frac{\partial \mathrm{TC}}{\partial Q}=\frac{-1}{Q^{2}}\left[\frac{(h+\pi) D^{2}}{2} E^{2}[L]+A D\right]+\frac{h}{2}, \\
\frac{\partial T C}{\partial r_{i}}=D\left[\sum_{i=1}^{n} h_{i}-h+\frac{(h+\pi) D}{Q} E[L]\right] \frac{\partial E[L]}{\partial r_{i}}+h_{i}
\end{gathered}
$$

After that, we deduce from formula (3) that $\lim _{\mathrm{Q} \longrightarrow \infty}(\partial \mathrm{TC}) /(\partial \mathrm{Q})=h / 2>0$ and $\lim _{\mathrm{Q} \longrightarrow 0^{+}}(\partial \mathrm{TC}) /(\partial \mathrm{Q})=$ $-\infty<0$. Moreover, it is easy to know $\partial^{2} \mathrm{TC} / \partial Q^{2} \geq 0$, so if only $\partial \mathrm{TC} / \partial Q=0$ and $Q \geq 0$, the extreme value of $\mathrm{TC}(Q, r)$ is unique.
We continue with the terms in formula (4). Here we define that the distribution function and probability density function of $\max _{1 \leq i \leq n}\left\{L_{i}\right\}$ are $G(x)$ and $g(x)$, respectively, which can be expressed as follows

$$
\begin{aligned}
G(x) & =P\left[\max _{1 \leq i \leq n}\left\{L_{i}\right\} \leq x\right]=\prod_{i=1}^{n} P\left[L_{i} \leq x\right] \\
& =\prod_{i=1}^{n} P\left[Y_{i}-\frac{r_{i}}{D} \leq x\right]=\prod_{i=1}^{n} F_{i}\left(\frac{r_{i}}{D}+x\right),
\end{aligned}
$$

$$
g(x)=\frac{d G(x)}{d x}=\sum_{i=1}^{n}\left[f_{i}\left(\frac{r_{i}}{D}+x\right) \prod_{j=1, j \neq i}^{n} F_{j}\left(\frac{r_{j}}{D}+x\right)\right] .
$$

By the definition of expectation on a random variable, we can calculate $E[L]$ :

$$
\begin{aligned}
E[L] & =\int_{0}^{\infty} x g(x) d x \\
& =\sum_{i=1}^{n} \int_{0}^{\infty} x f_{i}\left(\frac{r_{i}}{D}+x\right) \prod_{j=1, j \neq i}^{n} F_{j}\left(\frac{r_{j}}{D}+x\right) d x
\end{aligned}
$$

In order to get $\partial \mathrm{TC} / \partial r_{i}$, we need to compute the firstorder partial derivative of $E[L]$ with respect to $r_{i}$ :

$$
\begin{aligned}
\frac{\partial E[L]}{\partial r_{i}} & \\
= & \frac{\partial}{\partial r_{i}} \lim _{u \rightarrow \infty} \int_{0}^{u} x g(x) d x \\
& =\frac{\partial}{\partial r_{i}} \lim _{u \rightarrow \infty}\left\{[x G(x)]_{0}^{u}-\int_{0}^{u} G(x) d x\right\} \\
& =\frac{\partial}{\partial r_{i}} \lim _{u \rightarrow \infty}\left\{u G(u)-\int_{0}^{u} G(x) d x\right\} \\
& =\lim _{u \rightarrow \infty} \frac{\partial}{\partial r_{i}}\left\{u G(u)-\int_{0}^{u} G(x) d x\right\} \\
& =\lim _{u \rightarrow \infty}\left\{u \frac{\partial G(u)}{\partial r_{i}}-\int_{0}^{u} \frac{\partial G(x)}{\partial r_{i}} d x\right\} \\
& =\frac{1}{D} \lim _{u \rightarrow \infty}\left\{u f_{i}\left(\frac{r_{i}}{D}+u\right) \prod_{j=1, j \neq i}^{n} F_{j}\left(\frac{r_{j}}{D}+u\right)\right. \\
& =-\frac{1}{D} \int_{0}^{u} f_{i}\left(\frac{r_{i}}{D}+x\right) \prod_{j=1, j \neq i}^{n} F_{j}\left(\frac{r_{j}}{D}+x\right) d x . \\
&
\end{aligned}
$$


According to formulas (4) and (7), we can deduce

$$
\begin{aligned}
\lim _{r_{i} \rightarrow \infty} \frac{\partial \mathrm{TC}}{\partial r_{i}}= & D\left(\sum_{i=1}^{n} h_{i}-h+\frac{(h+\pi) D}{Q} E[L]\right) \\
& \times \lim _{r_{i} \rightarrow \infty} \frac{\partial E[L]}{\partial r_{i}}+h_{i} \\
= & h_{i}>0 .
\end{aligned}
$$

Similarly, if $E[L]>Q\left(h-\sum_{i=1}^{n} h_{i}\right) / D(h+\pi)$, we can get $\lim _{r_{i} \rightarrow 0^{+}} \partial T C / \partial r_{i}<0$ and $\partial^{2} T C / \partial r_{i}^{2} \geq 0$.

In summary, there must be a unique global optimal solution for $\mathrm{TC}(Q, r)$.

4.2. Model Solution. According to the above analysis, the optimal values $Q^{*}$ and $r_{i}^{*}$ are interacted, which implies that the simple application of first-order partial derivatives cannot ensure that we can get the two optimal values simultaneously. To solve this problem we will use $E[L]$ as an intermediary to make some appropriate changes on the objective function: firstly transform the original problem into a one-dimensional optimization problem and find the optimal solution of $E[L]$ for problem $(P)$, and then get the optimal values of $Q$ and $r_{i}$. $(P)$.

The following steps can be adopted to solve the problem

(1) Define $\Phi_{1}(z)$ and $\Phi_{2}(z)$, where $z=E[L]$, and formula (2) can be decomposed into the following two according to decision variables:

$$
\begin{aligned}
& \Phi_{1}(z) \equiv \min _{Q}\left[\varphi_{1}(Q) \equiv D\left(\sum_{i} h_{i}-h\right) z\right. \\
& \left.+\frac{(h+\pi) D^{2} z^{2}}{2 Q}+\frac{h}{2} Q+\frac{A D}{Q}\right], \\
& \text { s.t. } Q>0 \text {, } \\
& \Phi_{2}(z) \equiv \min _{r_{i}}\left[\varphi_{2}(r) \equiv-D \sum_{i=1}^{n} h_{i} E\left[L_{i}\right]\right] \text {, } \\
& \text { s.t. } E\left[L_{i}\right] \leq z, r_{i} \geq 0, i=1,2, \ldots, n \text {. }
\end{aligned}
$$

Since $\varphi_{1}(Q)$ is a simple convex function in $Q$, the optimal value is

$$
Q^{*}=\sqrt{\frac{(h+\pi) D^{2} z^{2}+2 A D}{h}}
$$

(2) Substitute $Q^{*}$ into $\Phi_{1}(z)$, and the optimal solution of the minimization problem can be expressed as a function in $z$ :

$$
\Phi_{1}(z)=D\left(\sum_{i} h_{i}-h\right) z+\frac{(h+\pi) D^{2} z^{2}}{2 Q^{*}}+\frac{h}{2} Q^{*}+\frac{A D}{Q^{*}}
$$

As $\partial^{2} \Phi_{1}(z) / \partial z^{2}=2 A(h+\pi) D^{3} / h Q^{* 3}>0$, we can know $\Phi_{1}(z)$ is a strict convex function in $z$, where $Q^{*}$ meets the constraint that it should be larger than 0 , then formula (9) can be regarded as a convex programming problem.

As $\varphi_{2}(r)=\sum_{i=1}^{n} h_{i} r_{i}-D \sum_{i=1}^{n} h_{i} E\left[Y_{i}\right]$ is a linear function in $r_{i}$, and $E[L]=E\left[\max _{i}\left\{Y_{i}-\left(r_{i} / D\right), 0\right\}\right]$ is a convex function in $r_{i}$, then formula (10) can be also regarded as a convex programming problem, which has a very good feature as shown in the next step.

(3) Define $z^{\prime \prime} \equiv \max _{r_{i}>0, i=1, \ldots, n} E[L]=E\left[\max _{i} Y_{i}\right] \cdot \varphi_{2}(r)$ increases with the gradual increase of $r_{i}$. At the same time, $E[L]$ decreases nonlinearly. So it can be inferred that $E[L]$ will increase to the maximum as $r_{i}$ decreases to 0 gradually. As a result, we can transform the constraint $r_{i} \geq 0$ into $E[L] \leq z^{\prime \prime}$, where $0 \leq z \leq z^{\prime \prime}$.

From the above analysis, $\mathrm{TC}(Q, r)$ can be decomposed into two functions in $Q$ and $r$ :

$$
\mathrm{TC}(Q, r)=\varphi_{1}(Q)+\varphi_{2}(r)
$$

Furthermore, the original problem $(P)$ can be transformed into the following problem $(R)$ :

$$
\min _{0 \leq z \leq z^{\prime \prime}}\left[\Phi_{1}(z)+\Phi_{2}(z)\right]
$$

where $z=E[L], z^{\prime \prime}=E\left[\max _{i} Y_{i}\right]$. As $\Phi_{1}(z)+\Phi_{2}(z)$ is a convex function, problem $(R)$ is a one-dimensional search problem about $z$ under the given constraint of $0 \leq z \leq z^{\prime \prime}$. We can use the one-dimensional search method to find all possible values of $z$ under the constraint and obtain the optimal solution of problem $(R)$, then get the optimal value of $Q^{*}$, and finally find $r_{i}^{*}$ by solving the equations.

Numerical Analysis. Assume that the Supply-Hub orders components from two suppliers, and the lead time $Y_{i}$ of the two components obeys exponential distribution with the parameter $\lambda_{i}(i=1,2)$, of which the probability density function is $f(x)=\lambda_{i} e^{-\lambda_{i} x}$, moreover $D=$ 250 units/year, $A=800$ units/year, $h=70$ USD/units $*$ year, $h_{1}=30$ USD/units $*$ year, $h_{2}=20$ USD/units $*$ year, $\pi=$ $400 \mathrm{USD} /$ product, and $\lambda_{1}=25, \lambda_{2}=20$.

Table 1 shows that the total cost decreases as the value of $z$ increases by 0.001 units from 0 . When $z=0.052$, the total cost reaches the minimum, and after that, it will be greater than the minimum again with the increase of $z$. Therefore, we obtain $z^{*}=0.052$ and $Q^{*}=82.75869$. Then by calculating the nonlinear programming of formula (10), we get $r_{1}^{*}=1.81699, r_{2}^{*}=5.23407$, and consequently $\mathrm{TC}^{*}(Q, r)=5718.469$.

As mentioned above, we only take costs of the SupplyHub and manufacture into consideration and finally prove that there must be an optimal assembly quantity $Q$ and an optimal reorder point $r_{i}$ of each component, which can contribute to the lowest cost $\mathrm{TC}^{*}(Q, r)$ under centralized decision making. Obviously, we also need to talk about the relations between the Supply-Hub and suppliers. In the next section, we will discuss how the Supply-Hub makes use of 
TABLE 1: Optimal solutions of the expected total cost.

\begin{tabular}{lcccc}
\hline$z$ & $Q^{*}$ & $\varphi_{1}(Q)$ & $\varphi_{2}(r)$ & $\mathrm{TC}(Q, r)$ \\
\hline 0.045 & 81.0189 & 5446.323 & 283.5354 & 5729.859 \\
0.046 & 81.25423 & 5457.796 & 268.9064 & 5726.703 \\
0.047 & 81.49403 & 5469.582 & 254.5106 & 5724.093 \\
0.048 & 81.73826 & 5481.678 & 240.3141 & 5721.992 \\
0.049 & 81.98688 & 5494.081 & 226.3121 & 5720.394 \\
0.05 & 82.23985 & 5506.789 & 212.493 & 5719.282 \\
0.051 & 82.49713 & 5519.799 & 198.8464 & 5718.646 \\
0.052 & 82.75869 & 5533.108 & 185.3613 & 5718.469 \\
0.053 & 83.02447 & 5546.713 & 172.0275 & 5718.74 \\
0.054 & 83.29444 & 5560.611 & 158.8353 & 5719.446 \\
0.055 & 83.56857 & 5574.8 & 145.775 & 5720.575 \\
\hline
\end{tabular}

Punishment and Reward mechanisms to coordinate each supplier and reaches its goal of the expected service level.

\section{Collaborative Replenishment Mechanism Based on Punishment and Reward}

The above discussion shows that in ATO systems, the uncertainty of suppliers' delivery lead time will inevitably lead to the occurrence of shortages. If the Supply-Hub and suppliers both focus on the elimination of low efficiency, the shortage cost of the Supply-Hub will be higher than suppliers. In this sense, it implies that the Supply-Hub has a higher concern for shortages than suppliers. At the same time, compared with endeavoring to avoid shortages, suppliers are more willing to realize the overall optimization of supply chain with the premise of adding their own profits. Therefore, it is necessary for the Supply-Hub to impose punishment and reward incentives on suppliers, by which we can not only reduce the uncertainty but also increase the efficiency of supply chain.

This part will establish ordering relations between suppliers and the Supply-Hub based on BOM and will explore how to achieve the expected service level with the application of punishment and reward mechanisms. The implementation of the mechanisms can be described as that: if the supplier delay in delivery for a period of $t$, the Supply-Hub will punish him with a penalty of $P_{t}$, and if the supplier deliver on time, he will get a bonus of $B$. By calculating the Hessian matrix, we can verify the convexity of objective function and get the optimal values of decision variables under the given punishment and reward factors. The relations between the service level of the Supply-Hub and the two factors will be shown in figures.

The lead time of supplier $i$ is a random variable $Y_{i}$, and we assume $Y_{i}$ follows the uniform distribution in the range of $\left[u_{i}-R_{i}, u_{i}+R_{i}\right]$ with mean value $u=u_{i}$, and variance $\delta=R_{i}^{2} / 3, i=1,2, \ldots, n$. Then the service level of the SupplyHub is the probability that $n$ suppliers deliver on time at the same time, which is

$$
\rho=\prod_{i=1}^{n}\left(1-\frac{\left(u_{i}+R_{i}\right)-r_{i} / D}{2 R_{i}}\right) .
$$

As we know, the variance of actual lead time can be reduced by increasing investment and improving inventory level of raw materials. Here we assume that the investment of reducing the variance of actual lead time to zero is $\theta_{i}$, and each supplier will only try to reduce unit variance, so the investment cost for supplier $i$ is $C(\delta)=\theta_{i} / \delta=3 \theta_{i} / R_{i}^{2}$.

5.1. Punishment Coordination Mechanism. We now assume that the Supply-Hub implements the same punishment and reward mechanisms to every supplier.

For supplier $i$, the expected total cost is the sum of holding cost of the component, penalty and investment cost of reducing lead time variance, which is

$$
C\left(r_{i}, R_{i}\right)=h_{i} E\left[L_{i}^{-}\right]+P E\left[L_{i}^{+}\right]+\frac{3 \theta_{i}}{R_{i}^{2}}, \quad i=1,2, \ldots, n,
$$

where

$$
\begin{aligned}
E\left[L_{i}^{-}\right] & =\int_{u_{i}-R_{i}}^{r_{i} / D}\left(\frac{r_{i}}{D}-x\right) f_{i}(x) d x \\
& =\left[\frac{r_{i}}{2 D}+\frac{r_{i}^{2}}{4 D^{2} R_{i}}+\frac{R_{i}}{4}-\frac{u_{i}}{2}-\frac{r_{i} u_{i}}{2 D R_{i}}+\frac{u_{i}^{2}}{4 R_{i}}\right], \\
E\left[L_{i}^{+}\right] & =\int_{r_{i} / D}^{u_{i}+R_{i}}\left(x-\frac{r_{i}}{D}\right) f_{i}(x) d x \\
& =\left[-\frac{r_{i}}{2 D}+\frac{r_{i}^{2}}{4 D^{2} R_{i}}+\frac{R_{i}}{4}+\frac{u_{i}}{2}-\frac{r_{i} u_{i}}{2 D R_{i}}+\frac{u_{i}^{2}}{4 R_{i}}\right] .
\end{aligned}
$$

For convenience, we replace the expected lead time $r_{i} / D$ with $A_{i}$, then the cost function of supplier $i$ can be expressed as

$$
\begin{aligned}
C\left(r_{i}, R_{i}\right)= & h_{i}\left[\frac{A_{i}}{2}+\frac{A_{i}^{2}}{4 R_{i}}+\frac{R_{i}}{4}-\frac{u_{i}}{2}-\frac{A_{i} u_{i}}{2 R_{i}}+\frac{u_{i}^{2}}{4 R_{i}}\right] \\
& +P\left[-\frac{A_{i}}{2}+\frac{A_{i}^{2}}{4 R_{i}}+\frac{R_{i}}{4}+\frac{u_{i}}{2}-\frac{A_{i} u_{i}}{2 R_{i}}+\frac{u_{i}^{2}}{4 R_{i}}\right]+\frac{3 \theta}{R_{i}^{2}} .
\end{aligned}
$$

Take the first-order derivatives of cost function $C\left(r_{i}, R_{i}\right)$ with respect to $A_{i}$ and $R_{i}$, respectively, and make them equal to 0 as follows.

$$
\frac{\partial C_{i}}{\partial A_{i}}=\frac{h_{i}}{2 R_{i}}\left(R_{i}+A_{i}-u_{i}\right)+\frac{P}{2 R_{i}}\left(-R_{i}+A_{i}-u_{i}\right)=0,
$$

where

$$
A_{i}(P)=\frac{\left(P-h_{i}\right) R_{i}}{\left(P+h_{i}\right)}+u_{i}
$$

Similarly,

$$
\begin{aligned}
\frac{\partial C_{i}}{\partial R_{i}}= & \frac{h_{i}}{4 R_{i}^{2}}\left(R_{i}^{2}-A_{i}^{2}+2 A_{i} u_{i}-u_{i}^{2}\right) \\
& +\frac{P}{4 R_{i}^{2}}\left(R_{i}^{2}-A_{i}^{2}+2 A_{i} u_{i}-u_{i}^{2}\right)-\frac{6 \theta_{i}}{R_{i}^{3}}=0
\end{aligned}
$$


Substitute $A_{i}(p)$ into the above formula, we can get

$$
R_{i}(P)=\left[\frac{6 \theta_{i}\left(h_{i}+P\right)^{2}}{h_{i} P}\right]^{1 / 3} .
$$

Then substitute $R_{i}(P)$ into formula (20)

$$
A_{i}(p)=\frac{\left(P-h_{i}\right)\left[6 \theta_{i}\left(h_{i}+P\right)^{2} / h_{i} P\right]^{1 / 3}}{\left(P+h_{i}\right)}+u_{i} .
$$

After calculation, Hessian matrix of the binary differentiable function is

$$
\begin{gathered}
\left|\begin{array}{cc}
\frac{h_{i}+P}{2 R_{i}} & \frac{\left(h_{i}+P\right)\left(u_{i}-A_{i}\right)}{2 R_{i}^{2}} \\
\frac{\left(h_{i}+P\right)\left(u_{i}-A_{i}\right)}{2 R_{i}^{2}} & \frac{\left(h_{i}+P\right)\left(A_{i}-u_{i}\right)^{2}}{2 R_{i}^{3}}+\frac{18 \theta_{i}}{R_{i}^{4}}
\end{array}\right| \\
=\frac{9 \theta_{i}\left(h_{i}+P\right)}{R_{i}^{5}}>0 .
\end{gathered}
$$

So $C\left(r_{i}, R_{i}\right)$ is a convex function, and we can know $A_{i}(P)$ and $R_{i}(P)$ are the optimal values when $P$ is given.

Based on the above analyses, substitute $A_{i}(P)$ and $R_{i}(P)$ into the expression of $\rho$, and the service level of the SupplyHub under the given punishment factor $P$ can be obtained:

$$
\begin{aligned}
\rho & =\prod_{i=1}^{n}\left(1-\frac{\left(u_{i}+R_{i}\right)-r_{i} / D}{2 R_{i}}\right) \\
& =\prod_{i=1}^{n}\left(1-\frac{\left(u_{i}+R_{i}\right)-A_{i}}{2 R_{i}}\right) \\
& =\prod_{i=1}^{n} \frac{P}{h_{i}+P} .
\end{aligned}
$$

As $\partial \rho / \partial P=\sum_{i=k}^{n}\left(h_{k} /\left(h_{k}+P\right)^{2}\right) \prod_{i \neq k}^{n} P /\left(h_{i}+P\right)>$ 0 , the expected service level is an increasing function in punishment factor $P$, and only when $P \longrightarrow \infty, \rho \longrightarrow 1$, which means when the punishment factor is large enough, the service level will approach illimitably to $100 \%$. In fact, the conclusion is in line with the practical situation. If the punishment factor is very large, the supplier's late delivery will lead to a significant increase of total cost, thus suppliers will avoid delay delivery.

Numerical Analysis. We assume that the Supply-Hub places orders, respectively, to two suppliers. Here we follow the parameters in previous chapter, $h_{1}=30 \mathrm{USD} /$ unit $*$ year and $h_{2}=20$ USD/unit $*$ year. A relational diagram between the expected service level of the Supply-Hub $\rho$ and the value of punishment factor $P$ can be illustrated in Figure 5 .

5.2. Reward Coordination Mechanism. When the SupplyHub uses reward mechanism to coordinate the JIT operation, for supplier $i$, the cost function is the sum of holding cost of

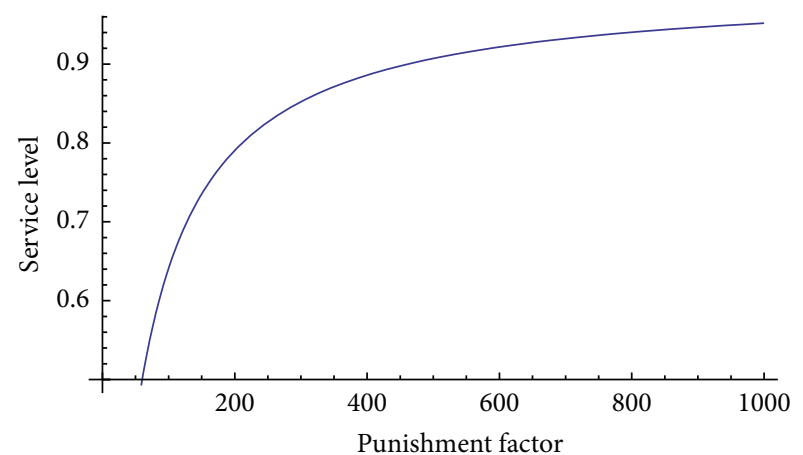

FIGURE 5: Relation between expected service level and punishment factor.

the component, bonus and actual investment cost of reducing lead time variance, which is

$$
\begin{aligned}
C\left(r_{i}, R_{i}\right)= & h_{i} E\left[L_{i}^{-}\right]-B \cdot P\left(u_{i}-R_{i} \leq Y_{i} \leq A_{i}\right) \\
& +\frac{3 \theta_{i}}{R_{i}^{2}}, \quad i=1,2, \ldots, n .
\end{aligned}
$$

Similarly, take the first-order derivatives of cost function $C\left(r_{i}, R_{i}\right)$ with respect to $A_{i}$ and $R_{i}$, respectively, and make them equal to 0 , then we can get the expressions of $A_{i}$ and $R_{i}$

$$
\begin{gathered}
A_{i}(B)=\frac{B}{h_{i}}-\frac{24 h_{i} \theta_{i}}{B^{2}}+u_{i}, \\
R_{i}(B)=\frac{24 h_{i} \theta_{i}}{B^{2}} .
\end{gathered}
$$

After calculation, Hessian matrix of the binary differentiable function is

$$
\begin{gathered}
\left|\begin{array}{cc}
\frac{h_{i}}{2 R_{i}} & \frac{h_{i}\left(u_{i}-A_{i}\right)+B}{2 R_{i}^{2}} \\
\frac{h_{i}\left(u_{i}-A_{i}\right)+B}{2 R_{i}^{2}} & \frac{h_{i}\left(A_{i}-u_{i}\right)^{2}-2 B\left(A_{i}-u_{i}\right)}{2 R_{i}^{3}}+\frac{18 \theta_{i}}{R_{i}^{4}}
\end{array}\right| \\
=\frac{B^{2}}{8 R_{i}^{4}}>0 .
\end{gathered}
$$

So $C\left(r_{i}, R_{i}\right)$ is a convex function, then we can know $A_{i}(B)$ and $R_{i}(B)$ are the optimal values if $B$ is given.

Based on the above analyses, substitute $A_{i}(B)$ and $R_{i}(B)$ into the expression of $\rho$, we can get the service level of the Supply-Hub under the given $B$ :

$$
\begin{aligned}
\rho & =\prod_{i=1}^{n}\left(1-\frac{\left(u_{i}+R_{i}\right)-r_{i} / D}{2 R_{i}}\right) \\
& =\prod_{i=1}^{n}\left(1-\frac{\left(u_{i}+R_{i}\right)-A_{i}}{2 R_{i}}\right) \\
& =\prod_{i=1}^{n} \frac{B^{3}}{48 \theta_{i} h_{i}^{2}} .
\end{aligned}
$$


TABLE 2: Corresponding reward factor $B$ and expected service level obtained.

\begin{tabular}{lccccc}
\hline$B$ & 270.00 & 263.80 & 257.43 & 250.89 & 244.15 \\
\hline Service level & 100.00 & 95.00 & 90.00 & 85.00 & 80.00 \\
\hline
\end{tabular}

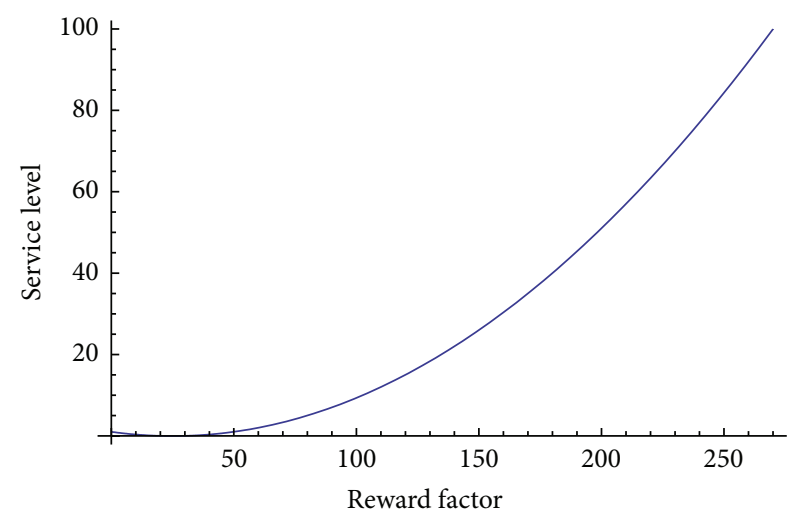

FIGURE 6: Relation between expected service level and reward factor.

It is easy to see that $\rho$ is an increasing function in $B$. That is to say, when the bonus is great enough, suppliers will do their best to delivery on time.

Numerical Analysis. Here we assume that the Supply-Hub still places orders, respectively, to two suppliers, with $h_{1}=$ 30 USD/unit*year and $h_{2}=20 \mathrm{USD} /$ unit $*$ year. The expected service level under corresponding reward factor can be calculated by Mathematica software, as shown in Table 2 .

In the table above, when the reward factor is 270.00 , the service level gets $100 \%$, which means the bonus that exceeds 270.00 is redundant. To illustrate the changing trend of expected service level $\rho$ caused by the changes of the value of $B$ better, a diagram can be drawn as Figure 6, in which the horizontal axis represents $B$, and the vertical axis stands for the expected service level $\rho$.

\section{Conclusion}

This paper constructs a collaborative replenishment model in the ATO system based on the Supply-Hub with delivery uncertainty. We transform the traditional model into a onedimensional optimization problem and derive the optimal assembly quantity and the optimal reorder point of each component. In order to enable collaborative replenishment, punishment and reward mechanisms are proposed for the Supply-Hub to coordinate the supply chain operation. The results show that if the punishment factor is very large, suppliers will avoid late delivery, also, if the reward factor is great enough, they will do their best to delivery on time. The numerical analysis also finds that punishment and reward mechanisms can significantly improve the suppliers' initiatives of collaborative replenishment, thereby leading to a higher service level in ATO systems. Overall, this paper provides a theoretical basis and also the useful guidance to the practice of collaborative replenishment in ATO systems based on the Supply-Hub with delivery uncertainty.

\section{Conflict of Interests}

The authors declare that there is no conflict of interests regarding the publication of this paper.

\section{Acknowledgments}

This work was supported by the National Natural Science Foundation of China (nos. 71102174, 71372019 and 71072035), Beijing Natural Science Foundation of China (nos. 9123028 and 9102016), Specialized Research Fund for Doctoral Program of Higher Education of China (no. 002020111101120019), Beijing Philosophy and Social Science Foundation of China (no. 11JGC106), Beijing Higher Education Young Elite Teacher Project (no. YETP1173), Program for New Century Excellent Talents in University of China (nos. NCET-10-0048 and NCET-10-0043), and Postdoctoral Science Foundation of China (2013M542066).

\section{References}

[1] G. Li, M. Q. Liu, Z. H. Wang, and B. Z. Peng, "Supply coordinating based on bonus policy in assembly under uncertain delivery time," Chinese Journal of Mechanical Engineering, vol. 26, no. 2, pp. 293-303, 2013.

[2] J.-S. Song, C. A. Yano, and P. Lerssrisuriya, "Contract assembly: dealing with combined supply lead time and demand quantity uncertainty," Manufacturing \& Service Operations Management, vol. 2, no. 3, pp. 287-296, 2000.

[3] J.-S. Song and D. D. Yao, "Performance analysis and optimization of assemble-to-order systems with random lead times," Operations Research, vol. 50, no. 5, pp. 889-918, 2002.

[4] Y. Lu, J.-S. Song, and D. D. Yao, "Order fill rate, leadtime variability, and advance demand information in an assemble-toorder system," Operations Research, vol. 51, no. 2, pp. 292-308, 2003.

[5] Y. Lu and J.-S. Song, "Order-based cost optimization in assemble-to-order systems," Operations Research, vol. 53, no. 1, pp. 151-169, 2005.

[6] V. N. Hsu, C. Y. Lee, and K. C. So, “Optimal component stocking policy for assemble-to-order systems with lead-time-dependent component and product pricing," Management Science, vol. 52, no. 3, pp. 337-351, 2006.

[7] S. H. Xu and Z. Li, "Managing a single-product assembleto-order system with technology innovations," Management Science, vol. 53, no. 9, pp. 1467-1485, 2007.

[8] E. L. Plambeck and A. R. Ward, "Note: a separation principle for a class of assemble-to-order systems with expediting," Operations Research, vol. 55, no. 3, pp. 603-609, 2007.

[9] E. L. Plambeck, "Asymptotically optimal control for an assemble-to-order system with capacitated component production and fixed transport costs," Operations Research, vol. 56, no. 5, pp. 1158-1171, 2008.

[10] J. Li and Y. Wang, "Supplier competition in decentralized assembly systems with price-sensitive and uncertain demand," Manufacturing \& Service Operations Management, vol. 12, no. 1, pp. 93-101, 2010. 
[11] Y. Lu, J.-S. Song, and Y. Zhao, "No-Holdback allocation rules for continuous-time assemble-to-order systems," Operations Research, vol. 58, no. 3, pp. 691-705, 2010.

[12] M. K. Doğru, M. I. Reiman, and Q. Wang, "A stochastic programming based inventory policy for assemble-to-order systems with application to the W model," Operations Research, vol. 58, no. 4, pp. 849-864, 2010.

[13] K. M. R. Hoena, R. Güllüb, G. J. van Houtuma, and I. M. H. Vliegenc, "A simple and accurate approximation for the order fill rates in lost-sales ATO systems," International Journal of Production Economics, vol. 133, no. 1, pp. 95-104, 2011.

[14] F. Bernstein, G. A. DeCroix, and Y. Wang, "The impact of demand aggregation through delayed component allocation in an assemble-to-order system," Management Science, vol. 57, no. 6, pp. 1154-1171, 2011.

[15] M. I. Reiman and Q. Wang, "A stochastic program based lower bound for assemble-to-order inventory systems," Operations Research Letters, vol. 40, no. 2, pp. 89-95, 2012.

[16] A. Bušić, I. Vliegen, and A. Scheller-Wolf, "Comparing Markov chains: aggregation and precedence relations applied to sets of states, with applications to assemble-to-order systems," Mathematics of Operations Research, vol. 37, no. 2, pp. 259-287, 2012.

[17] G. Li, L. Ran, X. Yue, and Z. Wang, "Dynamic pricing and supply coordination with reimbursement contract under random yield and demand," Discrete Dynamics in Nature and Society, vol. 2013, Article ID 631232, 10 pages, 2013.

[18] G. A. Decroix, J.-S. Song, and P. H. Zipkin, "Managing an assemble-to-order system with returns," Manufacturing \& Service Operations Management, vol. 11, no. 1, pp. 144-159, 2009.

[19] K. Zimmer, "Supply chain coordination with uncertain just-intime delivery," International Journal of Production Economics, vol. 77, no. 1, pp. 1-15, 2002.

[20] H. Gurnani, "Optimal lot-sizing policy with incentives for yield improvement," IEEE Transactions on Semiconductor Manufacturing, vol. 18, no. 2, pp. 304-308, 2005.

[21] H. Gurnani and Y. Gerchak, "Coordination in decentralized assembly systems with uncertain component yields," European Journal of Operational Research, vol. 176, no. 3, pp. 1559-1576, 2007.

[22] H. Gurnani and M. Shi, "A bargaining model for a firsttime interaction under asymmetric beliefs of supply reliability," Management Science, vol. 52, no. 6, pp. 865-880, 2006.

[23] E. Barnes, J. Dai, S. Deng et al., "On the strategy of supply-hubs for cost reduction and responsiveness," The Logistics InstituteAsia Pacific Report, 2000.

[24] J. Shah and M. Goh, "Setting operating policies for supply hubs," International Journal of Production Economics, vol. 100, no. 2, pp. 239-252, 2006.

[25] S. H. Ma and F. M. Gong, "Collaborative decision of distribution lot-sizing among suppliers based on supply-hub," Industrial Engineering and Management, vol. 14, no. 2, pp. 1-9, 2009.

[26] H. M. Gui and S. H. Ma, "A study on the multi-source replenishment model and coordination lot size decision-making based on supply-hub," Chinese Journal of Management Science, vol. 18, no. 1, pp. 78-82, 2010.

[27] G. Li, F. Lv, and X. Guan, "Collaborative scheduling model for supply-hub with multiple suppliers and multiple manufacturers," The Scientific World Journal, vol. 2014, Article ID 894573, 13 pages, 2014.

[28] S. K. Goyal, "Note on: manufacturing cycle time determination for a multi-stage economic production quantity mode," Management Science, vol. 23, pp. 332-333, 1976. 


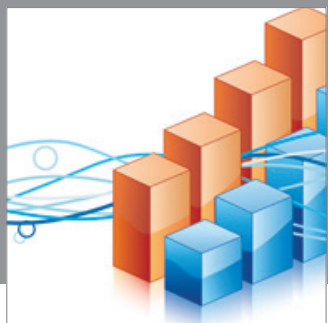

Advances in

Operations Research

mansans

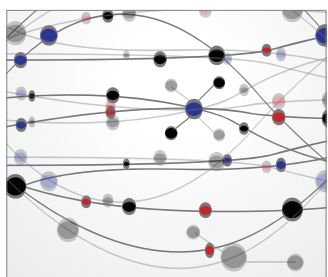

The Scientific World Journal
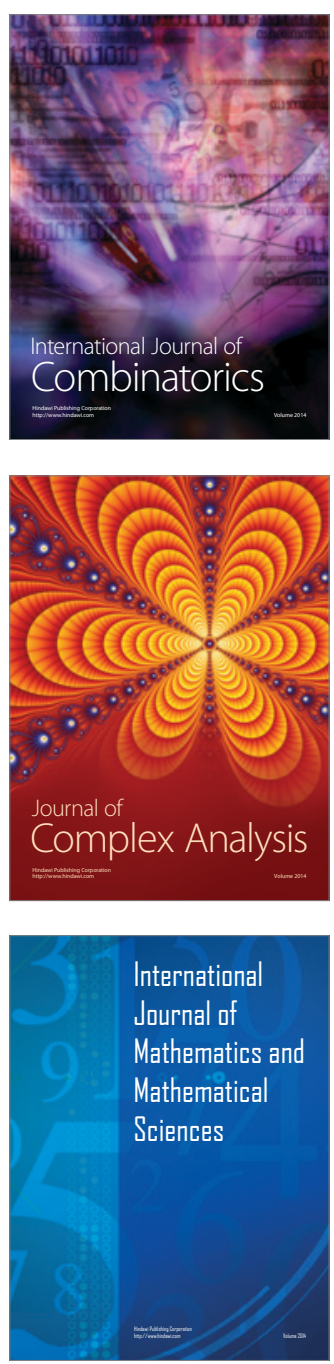
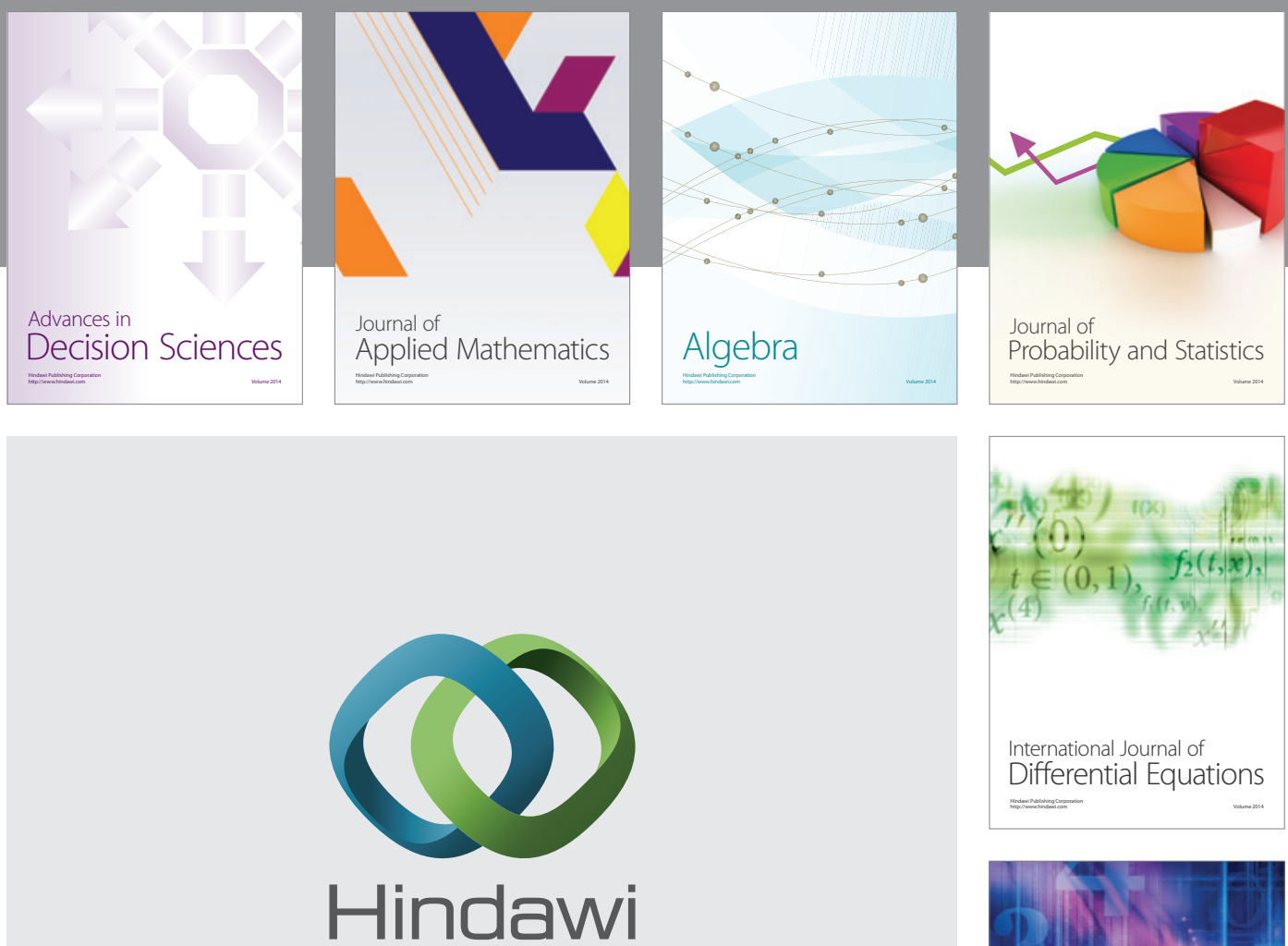

Submit your manuscripts at http://www.hindawi.com
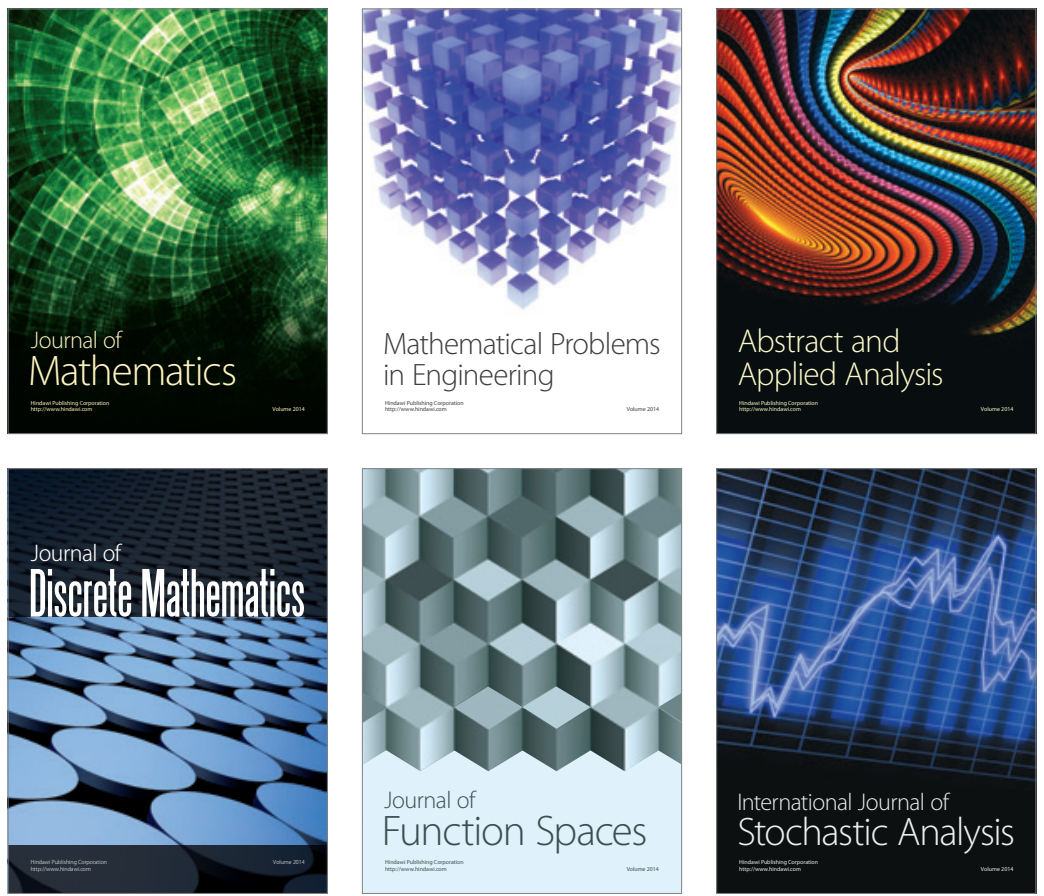

Journal of

Function Spaces

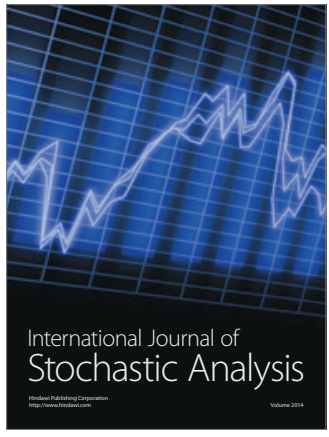

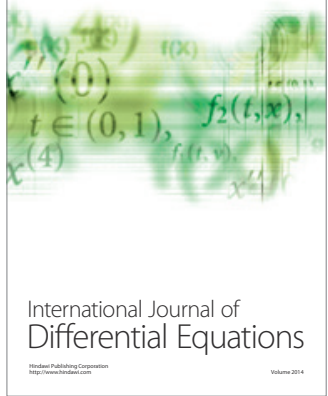
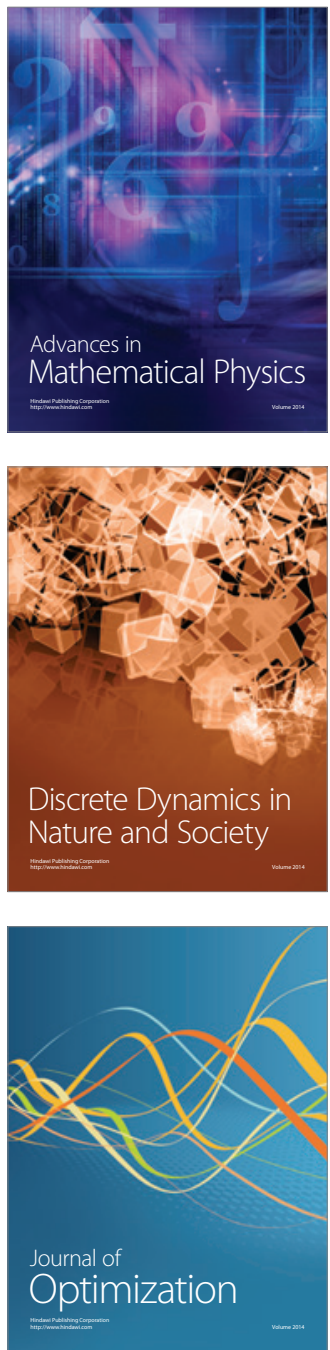